Universitext

For other titles in this series, go to

www.springer.com/series/223 
Marino Badiale $\cdot$ Enrico Serra

\section{Semilinear \\ Elliptic Equations \\ for Beginners}

Existence Results via

the Variational Approach

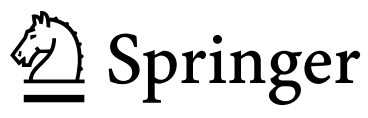




\author{
Marino Badiale \\ Dipartimento di Matematica \\ Università di Torino \\ Torino, Italy \\ marino.badiale@unito.it
}

\author{
Enrico Serra \\ Dipartimento di Matematica \\ Università di Torino \\ Torino, Italy \\ enrico.serra@polito.it
}

\author{
Editorial board: \\ Sheldon Axler, San Francisco State University, San Francisco, CA, USA \\ Vincenzo Capasso, Università degli Studi di Milano, Milan, Italy \\ Carles Casacuberta, Universitat de Barcelona, Barcelona, Spain \\ Angus J. Macintyre, Queen Mary, University of London, London, UK \\ Kenneth Ribet, University of California, Berkeley, CA, USA \\ Claude Sabbah, CNRS, École Polytechnique, Palaiseau, France \\ Endre Süli, University of Oxford, Oxford, UK \\ Wojbor Woyczynski, Case Western Reserve University, Cleveland, OH, USA
}

ISBN 978-0-85729-226-1

e-ISBN 978-0-85729-227-8

DOI 10.1007/978-0-85729-227-8

Springer London Dordrecht Heidelberg New York

British Library Cataloguing in Publication Data

A catalogue record for this book is available from the British Library

Mathematics Subject Classification (2000): 35-01, 35A15, 35B38, 35J15, 35J20, 35J25, 35J60, 35J61, $35 \mathrm{~J} 92$

(C) Springer-Verlag London Limited 2011

Apart from any fair dealing for the purposes of research or private study, or criticism or review, as permitted under the Copyright, Designs and Patents Act 1988, this publication may only be reproduced, stored or transmitted, in any form or by any means, with the prior permission in writing of the publishers, or in the case of reprographic reproduction in accordance with the terms of licenses issued by the Copyright Licensing Agency. Enquiries concerning reproduction outside those terms should be sent to the publishers.

The use of registered names, trademarks, etc., in this publication does not imply, even in the absence of a specific statement, that such names are exempt from the relevant laws and regulations and therefore free for general use.

The publisher makes no representation, express or implied, with regard to the accuracy of the information contained in this book and cannot accept any legal responsibility or liability for any errors or omissions that may be made.

Cover design: deblik

Printed on acid-free paper

Springer is part of Springer Science+Business Media (www.springer.com) 


\section{Preface}

This book is an introduction to the study of a wide class of partial differential equations, namely semilinear elliptic problems, through the application of variational methods and critical point theory. Semilinear elliptic equations arise in a variety of contexts in geometry, physics, mechanics, engineering and, more recently, in life sciences. None of these fields can be investigated without taking into account nonlinear phenomena, and variational methods, as a branch or an evolution of the Calculus of Variations, are almost entirely concerned with nonlinearity. The theory has attained a spectacular success in the last thirty-or-so years, reaching a high level of complexity and refinement, and its applications are scattered throughout thousands of research papers. Furthermore, some of the simplest methods within the variational approach are nowadays classical tools in the field of nonlinear differential equations as fixed point and monotonicity methods are. They have become, in other words, part of the toolbox that any researcher in the field is supposed to have at hand.

This poses a stimulating problem: how to teach variational methods and semilinear elliptic equations to upcoming generations of students. In our teaching experience, we have noticed a recurrent phenomenon. Up to a certain grade, students possess a rather general knowledge of basic principles, for instance in Functional Analysis. At the next step however they are often confronted with problems taken from real research. This creates a gap that students generally fill in by themselves, following a path of hard work that requires a great deal of commitment and selfdiscipline.

Both of us have been teaching courses on the topics collected in this book for a number of years in various Italian universities, and the didactic demand that we encountered was almost always located in the gap described above. We found that the many excellent existing books on variational methods and critical point theory (e.g. $[2,18,26,35,43,45,48])$, all of them deservedly well-known internationally, are sometimes problematic for classroom use, precisely because they are too complete, or too long, or too advanced.

Hence, the purpose of this book, derived directly from classroom experience, is that of providing a support to the students and the teacher engaged in a first course in semilinear elliptic equations. We have tried to write a textbook that matches what 
we normally write on the blackboard in class. This is why the topics are introduced gradually, and in certain cases some redundancy is purposely added, to stress the fundamental steps in the building of the theory. As a consequence, in this book the reader will not find any sophisticated results, or fancy applications, or fashionable examples, but rather a first overview on the subject, in a sort of "elliptic equations for the layman", where all the details are written down. Every abstract result is immediately put into action to show how it works to solve an elliptic problem, reflecting the fact that the theory was developed with the intent of treating ever larger classes of equations. We believe that in this way the reader will be able not only to have the tools at hand, but also to see how they really act when applied to concrete problems. In the applications, we limited ourselves to the discussion of Dirichlet boundary value problems. While this is certainly reductive, it provides nonetheless a unified thread throughout the book, and has the advantage of introducing the student to the most common type of boundary conditions. Other types of problems can then be understood with a minimal effort, and the existing literature provides extensions of all sorts for the interested reader.

The contents of this book require a general preparation in analysis, including some notions of Functional Analysis, and some knowledge of the most common function spaces, such as Lebesgue and Sobolev spaces. These are all notions usually taught in undergraduate courses throughout the world. In any case, whenever we quote a result without proving it, we provide precise references for consultation.

These notes are to be used by two possibly distinct categories of students: those who need to know how some types of nonlinear problems can be handled, and who need the basic working tools from the variational approach and critical point theory, as well as students who are oriented towards research in the field of nonlinear differential equations. Clearly if for the former this book may be the last they read on the subject, for the latter it should be just the first. We set the level of these notes between a graduate course and a first year $\mathrm{PhD}$ course. Mathematicians, physicists and engineers are the natural audience this book is addressed to.

The material is divided into four chapters. The first one is an introduction, and contains a review of differential calculus for functionals, with many examples, and a few basic facts from the linear theory that will be used throughout the book. Convexity arguments complete the discussion providing the first examples of existence theorems.

Chapter 2 introduces the fundamentals of minimization techniques, for it is wellknown that the simplest way to obtain a critical point of a functional is to look for a global extremum, which in most of the cases is a global minimum. However, for indefinite (unbounded) functionals, although global minimum points cannot exist, minimization techniques can still be profitably used by constraining the functional on a set where it is bounded from below. Typical examples of such sets are spheres or the Nehari manifold. The unifying feature of this chapter is the fact that all problems share some compactness properties that simplify the convergence arguments. A final section contains some examples of quasilinear problems, to show how the methods constructed for semilinear equations can be easily applied to more difficult problems. 
On the contrary, Chapter 3 is still devoted to minimization techniques, but this time the examples are all taken from problems that lack compactness. Typically this may happen when the domain on which the equation is studied is not bounded or when the growth of the nonlinearity reaches a critical threshold. The chapter discusses some of the most common ways to overcome these problems, that are often very hard to solve. Of course only some prototype cases are presented, which is in the spirit of these notes.

Chapter 4 introduces the reader to the more refined variational methods, where minimization is replaced by minimax procedures. The methods are first introduced in an abstract setting, so that the reader can dispose of a general principle that is suited to handling more sophisticated procedures, beyond the scope of this book. Then we concentrate on the two most popular and widespread results: the Mountain Pass Theorem and the Saddle Point Theorem, each discussed with applications to the specific problems that motivated them.

Each chapter is accompanied by a selection of exercises, some of them guided, to test the reader's ability to refine and put into action the techniques just discussed.

A short bibliographical note closes each chapter: the interested reader can find there suggestions for further reading, mainly taken from more advanced books or from the celebrated papers that first solved the problems discussed in the text.

Torino, Italy

Marino Badiale Enrico Serra 


\section{Contents}

1 Introduction and Basic Results $\ldots \ldots \ldots \ldots \ldots$

1.1 Motivations and Brief Historical Notes . . . . . . . . . . . . . 1

1.2 Notation and Preliminaries . . . . . . . . . . . . . . 5

1.2 .1 Function Spaces $\ldots \ldots \ldots \ldots$

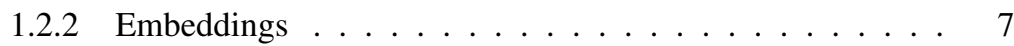

1.2 .3 Elliptic Equations . . . . . . . . . . . . . . 8

1.2.4 Frequently Used Results . . . . . . . . . . . . . . . . . . . 9

1.3 A Review of Differential Calculus for Real Functionals . . . . . . 11

1.3.1 Examples in Abstract Spaces _... . . . . . . . . . 15

1.3.2 Examples in Concrete Spaces . . . . . . . . . . . . . . 16

1.4 Weak Solutions and Critical Points . . . . . . . . . . . . 22

1.5 Convex Functionals . . . . . . . . . . . . . . . . 25

1.6 A Few Examples . . . . . . . . . . . . . . . . . . . . . . . 28

1.7 Some Spectral Properties of Elliptic Operators _ . . . . . . . . . 31

1.8 Exercises . . . . . . . . . . . . . . . . . . . 35

1.9 Bibliographical Notes . . . . . . . . . . . . . . . . . . 37

2 Minimization Techniques: Compact Problems . . . . . . . . . . . . . 39

2.1 Coercive Problems . . . . . . . . . . . . . . . . . . . . . . 39

2.2 A min-max Theorem . . . . . . . . . . . . . . . . 46

2.3 Superlinear Problems and Constrained Minimization . . . . . . . 55

2.3.1 Minimization on Spheres _. . . . . . . . . . . 56

2.3.2 Minimization on the Nehari Manifold . . . . . . . . . . . 59

2.4 A Perturbed Problem . . . . . . . . . . . . . . . . . . . . 63

2.5 Nonhomogeneous Nonlinearities . . . . . . . . . . . . . . . 74

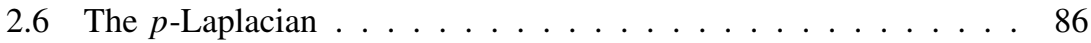

2.6.1 Basic Theory . . . . . . . . . . . . . . . . . . . . . 87

2.6 .2 Two Applications . . . . . . . . . . . . . . . . . . 91

2.7 Exercises . . . . . . . . . . . . . . . . . . . . 93

2.8 Bibliographical Notes . . . . . . . . . . . . . . . . . . . 95 
$3 \quad$ Minimization Techniques: Lack of Compactness _ . . . . . . . . . . 97

3.1 A Radial Problem in $\mathbb{R}^{N} \ldots \ldots$. . . . . . . . . . . . . . . . 97

3.2 A Problem with Unbounded Potential . . . . . . . . . . . . . . . . 104

3.3 A Serious Loss of Compactness . . . . . . . . . . . . . . . . . 107

3.4 Problems with Critical Exponent . . . . . . . . . . . . . . 119

3.4.1 The Prototype Problem . . . . . . . . . . . . . . 120

3.4.2 A Problem with a Radial Coefficient . . . . . . . . . . . 129

3.4 .3 A Nonexistence Result . . . . . . . . . . . . . . . . . . . 136

3.5 Exercises . . . . . . . . . . . . . . . . . 140

3.6 Bibliographical Notes . . . . . . . . . . . . . . . 143

4 Introduction to Minimax Methods . . . . . . . . . . . . . . . . . . . 145

4.1 Deformations . . . . . . . . . . . . . . . . . 146

4.2 The Minimax Principle . . . . . . . . . . . . . . . . . . . . 153

4.3 Two Classical Theorems . . . . . . . . . . . . . . . . . 155

4.4 Some Applications . . . . . . . . . . . . . . . . . . . . . . . 160

4.4.1 Superlinear Problems . . . . . . . . . . . . . . . 160

4.4.2 Asymptotically Linear Problems . . . . . . . . . . . . . . . 167

4.4.3 A Problem at Resonance . . . . . . . . . . . . . . . . . . 171

4.5 Problems with a Parameter . . . . . . . . . . . . . . . 178

4.6 Exercises . . . . . . . . . . . . . . . . 186

4.7 Bibliographical Notes . . . . . . . . . . . . . . . . 188

Index of the Main Assumptions . . . . . . . . . . . . . . . . . . . . . . . . 191

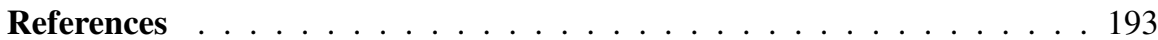

Index . . . . . . . . . . . . . . . . . . . . . . . . . 197 